The authors conclude that this study contributes to the growing body of literature suggesting a protective role for NSAIDs in PD. They call for further studies to elucidate the protective role such drugs might have in this disease.

Original article Wahner AD et al. (2007) Nonsteroidal antiinflammatory drugs may protect against Parkinson disease. Neurology 69: 1836-1842

\section{Short-term lesion dynamics on MRI predict longer-term disease activity in MS}

A group from Brigham and Women's Hospital in Boston, MA, has used mathematical modeling of MRI intensity change over time to examine the processes that lead to white matter damage in multiple sclerosis (MS) and their relationship with long-term disease progression.

Formulation of new lesions in MS is commonly characterized by an acute phase of blood-brain-barrier patency (active inflammation), as evidenced on contrast-enhanced MRI, followed by a chronic phase, demonstrated by signaling abnormalities on T1-weighted and T2-weighted MRI. This study revealed new T2 activity to last much longer than active inflammation (an average of 3-10 weeks, compared with an average of 2 weeks for the acute phase), representing an important noninflammatory (subacute) phase of lesion activity. Individual lesion characteristics were highly correlated with disease activity. Although the subacute phase was longer in larger lesions, smaller average peak lesion size showed a trend towards correlation with greater disability and greater residual damage. The amount of residual hyperintensity was positively correlated with the atrophy progression rate, and with level of disability. Furthermore, lesions in progressive MS were smaller and had a shorter duration of activity than those in relapsing-remitting MS, indicating an important relationship between lesion dynamics and clinical subtype.

These findings indicate that changes in the dynamics of individual lesions could represent a shift from inflammatory towards degenerative disease activity, and advancement towards disease progression. The authors suggest that there is the potential to stage progression of MS before the development of atrophy or disability.

Original article Meier DS et al. (2007) MR imaging intensity modeling of damage and repair in multiple sclerosis: relationship of short-term lesion recovery to progression and disability. AJNR Am J Neuroradiol 28: 1956-1963

\section{Brain tissue loss in early-stage multiple sclerosis predicts future cognitive decline}

Cognitive impairment occurs in $40-65 \%$ of patients with established multiple sclerosis (MS), and the psychosocial impact of dysfunction in verbal and visual recall, speed of information processing and abstract reasoning can be severe. MRI brain abnormalities, particularly atrophy, might precede cognitive dysfunction, and it has been suggested that early MRI scans could help identify which patients with MS are at greatest risk of cognitive decline.

To investigate this possibility, Summers et al. followed 30 patients with relapsing-remitting MS who had been recruited within 3 years of onset of their disease and who had been given an $\mathrm{MRI}$ scan within 1 year of diagnosis. At 5 years after diagnosis, all participants underwent neuropsychological assessment.

Attention span and speed of information processing deteriorated significantly in many of the participants over the 5-year follow-up: half showed a decline in IQ and a quarter had fullscale IQ decline. The rate of global brain atrophy apparent from the initial MRI scans correlated significantly with the difference in overall cognitive performance measured at 5 years. There was a particularly notable correlation between the rate at which atrophy occurred in earlystage disease and problems in memory and information processing at follow-up.

The authors conclude that neuroaxonal loss apparent from MRI scans taken shortly after diagnosis of MS can predict cognitive impairment later in the course of the disease.

Original article Summers MM et al. (2007) Cognitive impairment in relapsing-remitting multiple sclerosis can be predicted by imaging performed several years earlier. Mult Scler 13: 1-8 